University of Nebraska - Lincoln

DigitalCommons@University of Nebraska - Lincoln

December 2001

\title{
The biosynthetic gene cluster for the anticancer drug bleomycin from Streptomyces verticillus ATCC15003 as a model for hybrid peptide-polyketide natural product biosynthesis
}

\author{
B. Shen \\ University of California, Davis \\ Liangcheng Du \\ University of Nebraska - Lincoln, Idu3@unl.edu \\ C. Sanchez \\ University of California, Davis \\ D. J. Edwards \\ University of California, Davis \\ M. Chen \\ University of California, Davis \\ See next page for additional authors
}

Follow this and additional works at: https://digitalcommons.unl.edu/chemistrydu

Part of the Chemistry Commons

Shen, B.; Du, Liangcheng ; Sanchez, C.; Edwards, D. J.; Chen, M.; and Murrell, J. M., "The biosynthetic gene cluster for the anticancer drug bleomycin from Streptomyces verticillus ATCC15003 as a model for hybrid peptide-polyketide natural product biosynthesis" (2001). Liangcheng Du Publications. 8.

https://digitalcommons.unl.edu/chemistrydu/8

This Article is brought to you for free and open access by the Published Research - Department of Chemistry at DigitalCommons@University of Nebraska - Lincoln. It has been accepted for inclusion in Liangcheng Du Publications by an authorized administrator of DigitalCommons@University of Nebraska - Lincoln. 


\section{Authors}

B. Shen, Liangcheng Du, C. Sanchez, D. J. Edwards, M. Chen, and J. M. Murrell 


\title{
The biosynthetic gene cluster for the anticancer drug bleomycin from Streptomyces verticillus ATCC15003 as a model for hybrid peptide-polyketide natural product biosynthesis
}

\author{
B. Shen, L. Du, C. Sanchez, D.J. Edwards, M. Chen, and J.M. Murrell \\ Department of Chemistry, University of California, Davis, One Shields Avenue, Davis, CA 95616, USA
}

\begin{abstract}
The hybrid peptide-polyketide backbone of bleomycin (BLM) is assembled by the BLM megasynthetase that consists of both nonribosomal peptide synthetase (NRPS) and polyketide synthase (PKS) modules. BImIX/BImVIII/BImVII constitute a natural hybrid NRPS/PKS/NRPS system, serving as a model for both hybrid NRPS/PKS and PKS/NRPS systems. Sequence analysis and functional comparison of domains and modules of BImIX/BImVIII/BImVII with those of nonhybrid NRPS and PKS systems suggest that (1) the same catalytic sites appear to be conserved in both hybrid NRPS-PKS and nonhybrid NRPS or PKS systems, with the exception of the KS domains in the hybrid NRPS/PKS systems that are unique; (2) specific interpolypeptide linkers may play a critical role in intermodular communication to facilitate transfer of the growing intermediates between the interacting NRPS and/or PKS modules; and (3) posttranslational modification of the BLM megasynthetase has been accomplished by a single PPTase with a broad substrate specificity toward the apo forms of both acyl carrier proteins (ACPs) and peptidyl carrier proteins (PCPs).
\end{abstract}

Keywords: biosynthesis, bleomycin, hybrid peptide-polyketide, nonribosomal peptide synthetase, polyketide synthase, Streptomyces verticillus

Nonribosomal peptides and polyketides are two large families of natural products that include many clinically valuable drugs, such as vancomycin and erythromycin (antibacterial), cyclosporin and rapamycin (immunosuppressant), and bleomycin (BLM) and epothilone (antitumor). The biosynthesis of nonribosomal peptides and polyketides are catalyzed by nonribosomal peptide synthetases (NRPSs) and polyketide synthases (PKSs), respectively. NRPSs and PKSs apparently use a very similar strategy for the assembly of these two distinct classes of natural products by sequential condensation of amino acids and short carboxylic acids, respectively. Both NRPSs and type I PKSs are multifunctional proteins that are organized into modules. The number and order of modules on each NRPS or PKS protein determine the structural variations of the resulting peptide and polyketide products. Both systems use carrier proteins, peptidyl carrier protein (PCP) for NRPS and acyl carrier protein (ACP) for PKS, that are posttranslationally modified with the same 4'-phosphopantetheine prosthetic group to channel the growing peptide or polyketide intermediate during the elongation processes $[2-4,19,30]$. The latter modification is catalyzed by a family of 4'-phosphopantetheineyl transferases (PPTases) (Figure 1A) [20,31]. These striking structural and catalytic similarities between NRPSs and PKSs support the idea of combining individual NRPS and PKS modules into hybrid NRPS- PKS systems, in which an NRPS-bound growing peptidyl intermediate could be directly elongated by a PKS module (Figure 1B) or vice versa (Figure 1C) $[3,4,8,10]$. Since the modular architecture of both NRPSs and PKSs has been exploited successfully in the combinatorial biosyntheses of diverse "unnatural" natural products [2-4], it is imagined that hybrid NRPS-PKS systems, capable of incorporating both amino acids and short carboxylic acids into the final products, could lead to even greater chemical structural diversity.

We have been studying the biochemistry and genetics of BLM biosynthesis in Streptomyces verticillus ATCC15003 as a model for the biosynthesis of hybrid peptide-polyketide natural products $[6-10,23,24]$. Here we summarize our findings on (1) functional interactions between BLM NRPS and PKS modules that represent a natural hybrid NRPS-PKS system and (2) posttranslational modification of the BLM megasynthetase by the Svp PPTase that exhibits a broad substrate specificity toward both ACPs and PCPs. These results shed light on the molecular basis for a functional hybrid NRPSPKS system.

\section{BLM as an example of natural hybrid peptide- polyketide metabolites}

The BLMs, a family of anticancer antibiotics produced by S. verticillus, differ structurally at the C-terminal amines of the glycopeptides. The biosynthesis of BLMs has been extensively studied by feeding isotope-labeled precursors [12,2729]. These results unambiguously established the hybrid peptide-polyketide origin of the BLMs, the aglycone of which is derived from nine amino acids, an acetate, and two methyl groups derived from S-adenosyl methionine (AdoMet) (Figure 2A). Subsequent isolation and structural determination of a series of biosynthetic intermediates and shunt metabolites, including P-3A, P-4, P-5, and P-6m, from fermentation cultures led to the hypothesis of a processive pathway for assembly of the BLM hybrid peptide- polyketide backbone (Figure 2B). It is noteworthy that such a hybrid peptide-polyketide biosyn- 
(A) PPTase

\author{
(A) PPTase
} .<smiles>O[18O]</smiles>
apo-ACP
apo-PCP

\section{(B) NRPS/PKS}
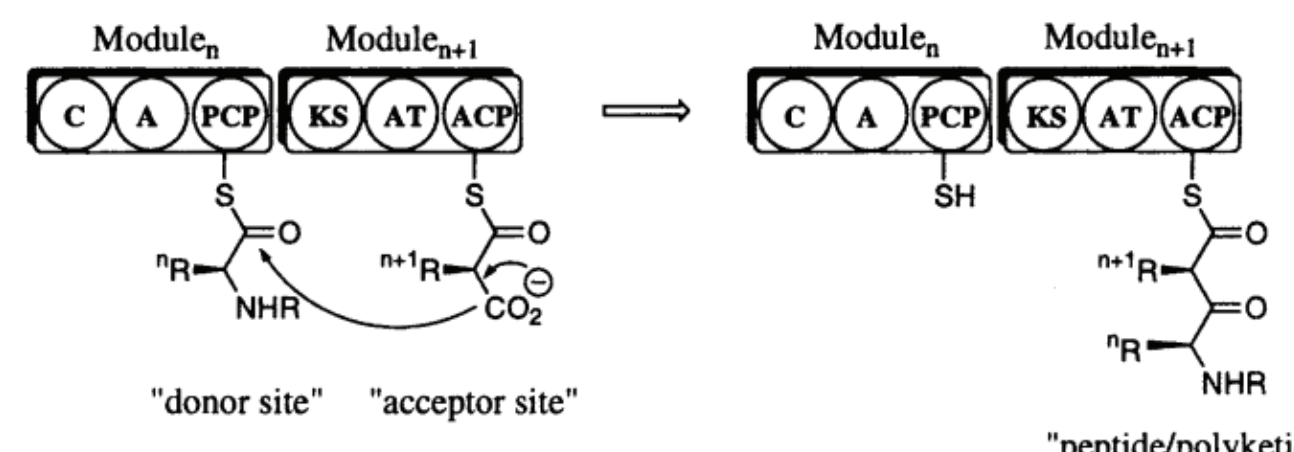

"peptide/polyketide"

\section{(C) PKS/NRPS}

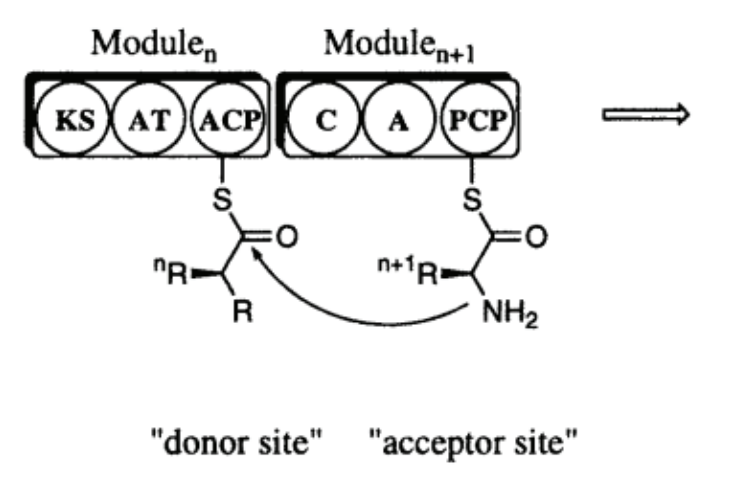

"polyketide/peptide"

Figure 1 (A) PPTase-catalyzed posttranslational modification of apo-ACP/PCP into holo-ACP/PCP. (B) Hybrid NRPS/PKS-catalyzed C-C bond formation in hybrid peptide/polyketide biosynthesis. (C) Hybrid PKS/ NRPS-catalyzed C-N bond formation in hybrid polyketide/peptide biosynthesis. These are hypothetical NRPS and PKS modules shown with only their respective core domains. Abbreviations for NRPS and PKS domains are: A, adenylation; ACP, acyl carrier protein; AT, acyltransferase; C, condensation; KS, ketoacyl synthase; and PCP, peptidyl carrier protein.

thesis model, inspired mainly by the fatty acid synthase, was put forward long before the characterization of the modular structure of either NRPS or PKS. According to today's hybrid NRPS-PKS model $[3,4,8,10]$, we could easily envisage biosynthesis of the BLM aglycone in three stages: (I) NRPS-mediated formation of P-3A from Ser, Asn, Asn, His, and Ala, (II) PKS-mediated elongation of P-3A by malonyl CoA and Adomet to yield P-4, and (III) NRPSmediated elongation of $\mathrm{P}-4$ by Thr to P-5 that is further elongated by $\beta$-Ala, Cys, and Cys to generate P-6m (Figure 2B). Therefore, the transition between stage I/II or II/III could potentially represent a natural hybrid NRPS/PKS (Figure 1B) or PKS/NRPS (Figure 1C) system, respectively.

\section{BLM megasynthetase as an example of natural hy- brid NRPS-PKS systems}

While the feeding experiments and the isolation of various biosynthetic and shunt metabolites motivated us to formulate the hybrid NRPS-PKS hypothesis for BLM biosynthesis, it is the cloning and characterization of the BLM biosynthetic gene cluster that provide direct evidence for the existence of the BLM megasynthetase, a natural hybrid NRPS-PKS system that catalyzes the assembly of the BLM aglycone backbone from the amino acid and short carboxylic acid precursors [6-10,23,24]. The blm gene cluster consists of 30 genes (Figure $3 \mathrm{~A}$ ), clustered with the $b \operatorname{lm} \mathrm{A}$ and $b \operatorname{lm} \mathrm{B}$ resistance genes that confer BLM resistance by drug sequestering and drug modification, respectively. Located in the center of the $\mathrm{blm}$ gene cluster are 10 NRPS genes encoding nine NRPS modules and a PKS gene encoding one PKS module. We predicted the substrate specificities of individual NRPS and PKS modules based on sequence analysis $[5,7,15,26]$ and subsequently confirmed the amino acid specificities of five NRPS modules and the malonyl CoA specificity of the PKS module biochemically in vitro (Figure 3A).

On the basis of the BLM structure and the deduced functions of individual NRPS and PKS domains and modules, we proposed a linear model for the BLM megasynthetase-templated assembly of the BLM peptide/polyketide/peptide agly- 
A
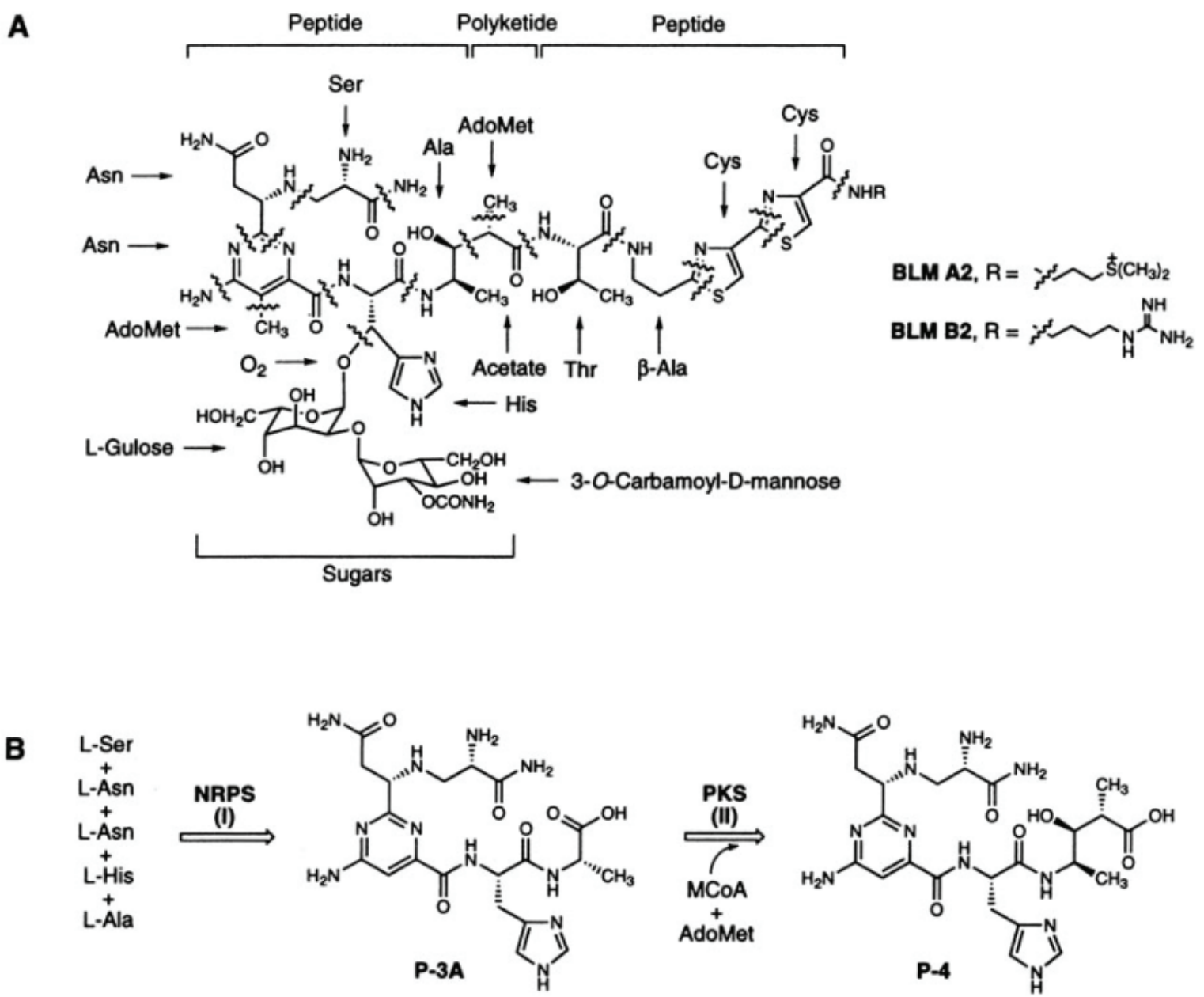<smiles>CC(NC(=O)[C@H](Cc1c[nH]cn1)NC(=O)c1cc(N)nc(C[C@H](CCNC(N)=O)NC[C@@H](N)C(N)=O)n1)C(C)C(=O)O</smiles>
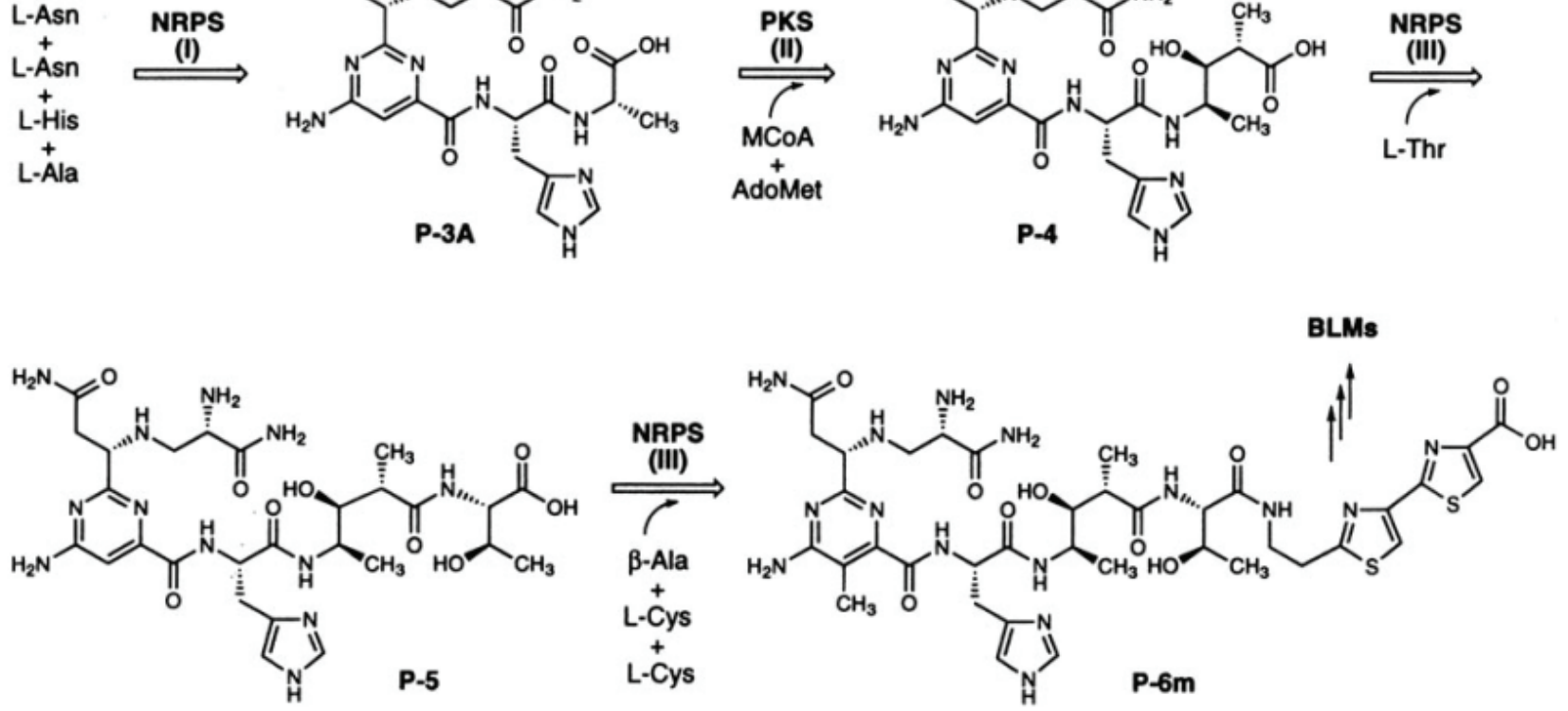

Figure 2 (A) Structures of BLMs and primary biosynthetic precursors for the BLM aglycone. (B) The proposed biosynthetic pathway for BLMs involved a hybrid NRPS/ PKS/ NRPS system. The growing hybrid peptide-polyketide intermediates P-3A, P-4, P-5, and P-6m were isolated from the wild-type $S$. verticillus fermentation and their structures were determined [12,27-29].

cone from nine amino acids and one acetate (Figure 3B) [7]. Individual modules are first primed with the amino acid or short carboxylic acid precursors, the choice of which is determined by the adenylation (A) domains of the NRPS modules or the acyltransferase (AT) domain of the PKS module, respectively (Figure $3 \mathrm{~A}$ ). The BlmVI, BlmV, BlmX, BlmIXm NRPS-mediated N-to-C directional assembly of the pseudopentapeptide intermediate proceeds by sequential condensation of Ser, Asn, Asn, His, and Ala, as evidenced by the isolation of P-3A (Figure 2B, stage I). In the next step, a malonate reacts with the resulting pentapeptide intermediate to form a $\beta$-ketothioester intermediate that is subsequently methylated at the $\beta$-position and reduced at the $\beta$-keto group, and this proposal is consistent with the isolation of P-4 (Figure 2B, stage II). The BlmVIII PKS module presumably dictates all these biosynthetic events and interacts with the upstream BlmIX NRPS module to channel the growing peptide intermediate from an NRPS module to a PKS module. After one cycle of polyketide elongation, peptide elongation is resumed by incorporation of a Thr residue. This step is catalyzed by the BlmVII NRPS module that interacts with the upstream BlmVIII PKS module to channel the growing polyketide intermediate 
A

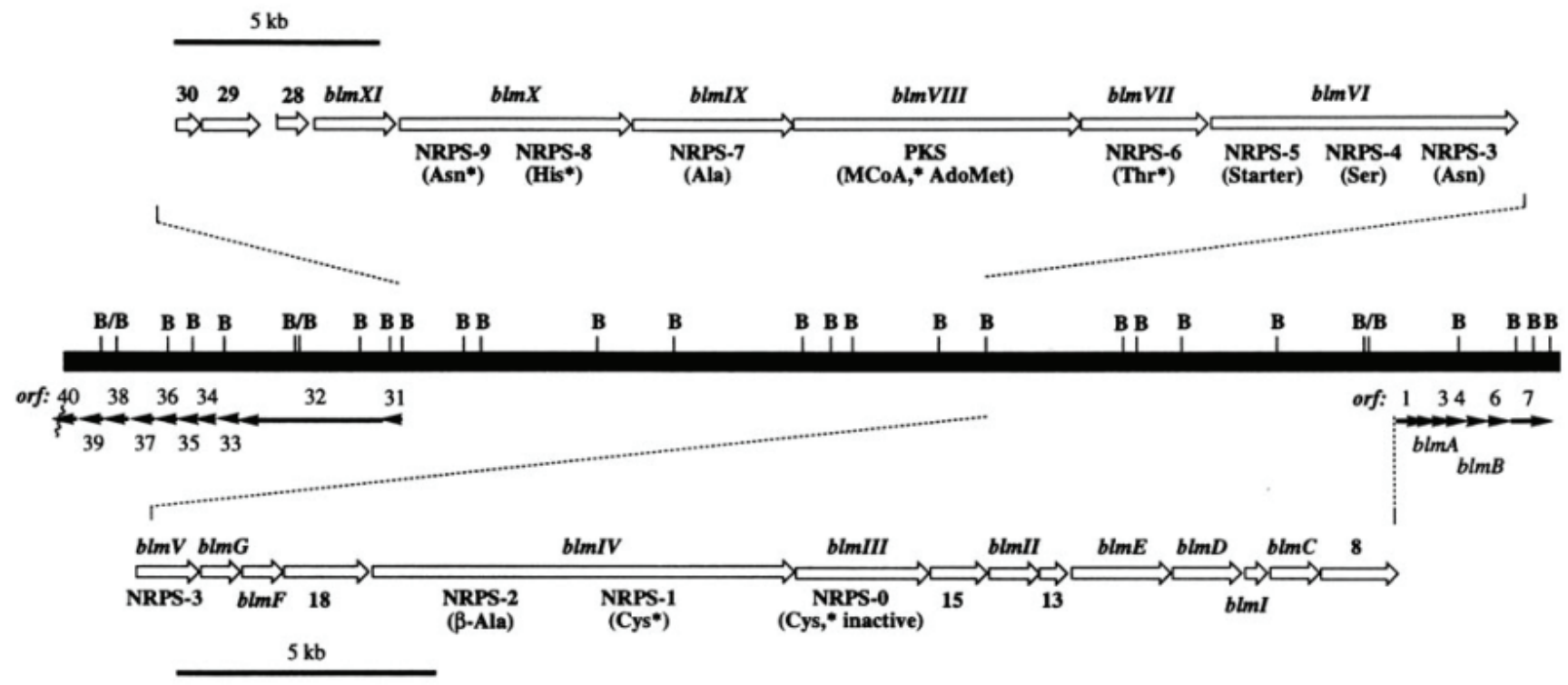

B

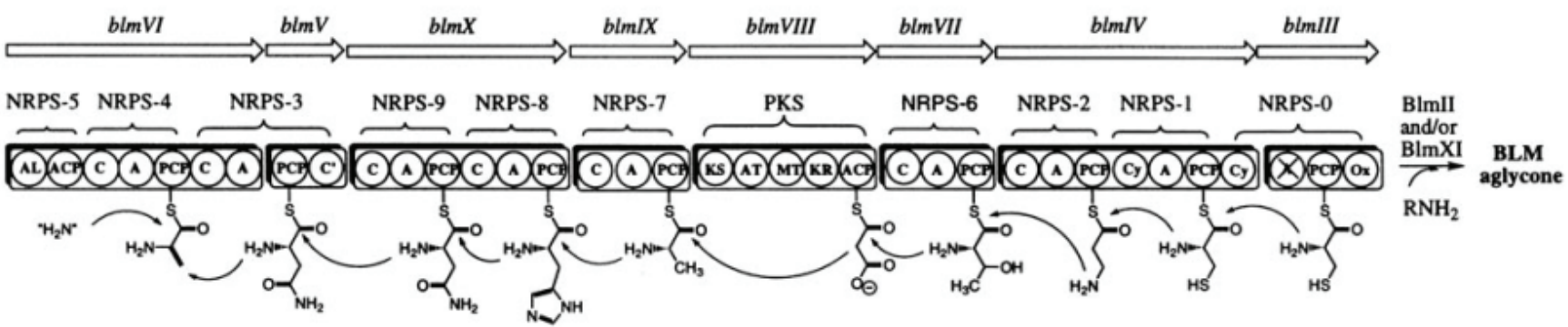

Figure 3 (A) Genetic organization of the BLM biosynthetic gene cluster [7]. Modules for individual NRPSand PKSare given along with their predicted or confirmed substrates $\left(^{*}\right)$ in parentheses. Three-letter amino acid designations were used. B, BamHI. (B) A linear model for the BLM megasynthetase-templated assembly of the BLM peptide/polyketide/peptide aglycone from nine amino acids and one acetate [7]. Abbreviations for NRPSand PKS domains are: A, adenylation; ACP, acyl carrier protein; AL, acyl CoA ligase; AT, aclytransferase; C and C', condensation; Cy, condensation/ cyclization; KR, ketoreductase; $\mathrm{KS}$, ketoacyl synthase; $\mathrm{MT}$, methyltransferase; Ox, oxidation; and PCP, peptidyl carrier protein. "NH${ }_{2}$," an unspecified amino group donor.

(as far as the active site is concerned) from a PKS module to an NRPS module. Subsequent elongation by three additional NRPS modules (BlmIV and BlmIII) incorporates $\beta$-Ala and two molecules of Cys into the BLM aglycone backbone (Figure $2 \mathrm{~B}$, stage III). Isolation of biosynthetic intermediates such as P-5 and P-6m supports the latter proposal. Finally, the fully assembled and modified BLM aglycone is released from the BLM megasynthetase complex via a nucleophilic substitution of the RCO-S-PCP species by an amine, RNH2. In contrast to most of the NRPSs and PKSs known to date that release the full-length peptide or polyketide intermediate from the enzyme complex by a thioesterase domain [2-4], BlmII and BlmXI, two discrete condensation enzymes identified within the blm gene cluster, have been proposed to catalyze the latter step [7]. Similar condensation (C) domain-catalyzed release of either RCO-S-PCP from NRPS [18] or RCO-S-ACP from PKS [17] has been proposed previously. The isolated biosynthetic intermediates, such as $\mathrm{P}-4, \mathrm{P}-5$, and $\mathrm{P}-5 \mathrm{~m}$, presumably resulted from premature departure from the BLM megasynthetase complex before the growing chain reaches its full length.

\section{Intermodular communication between BImIX/ BImVIII /BImVII as an example of both hybrid NRPS/ PKS and hybrid PKS/NRPS systems}

In peptide biosynthesis, the $\mathrm{C}-\mathrm{N}$ bond formation is mediated by the $\mathrm{C}$ domain that catalyzes nucleophilic substitution between the peptidyl-S-PCP from the upstream module, also known as "donor site" substrate, and the amino group of its cognate amino acyl-S-PCP, also known as "acceptor site" substrate $[1,25]$. In hybrid polyketide/peptide biosynthesis, however, the $\mathrm{C}$ domain of the hybrid PKS/NRPS mediates the same $\mathrm{C}-\mathrm{N}$ bond formation but by catalyzing the nucleophilic substitution between the acyl-S-ACP of the PKS module and the amino acyl-S-PCP of the NRPS (Figure 1C). This model suggests that the critical domains for functional interaction in a hybrid PKS/NRPS system should be the ACP and C do- 


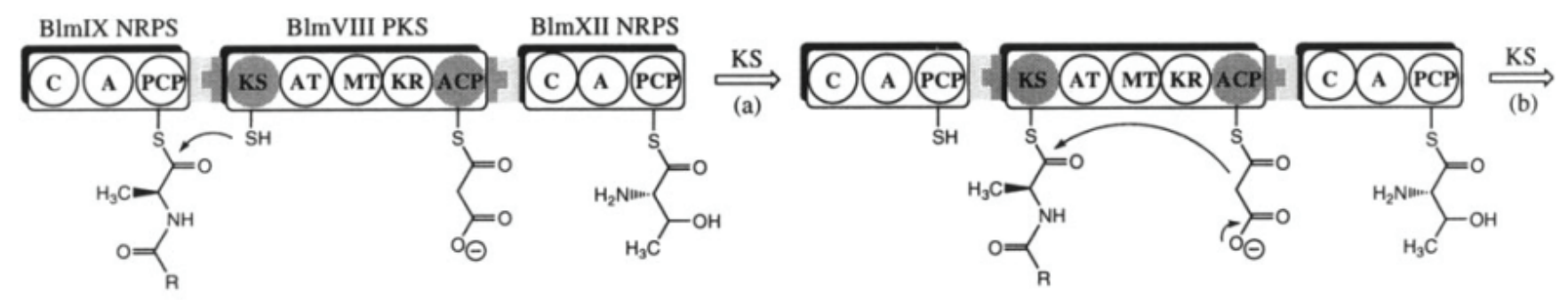

"peptide"
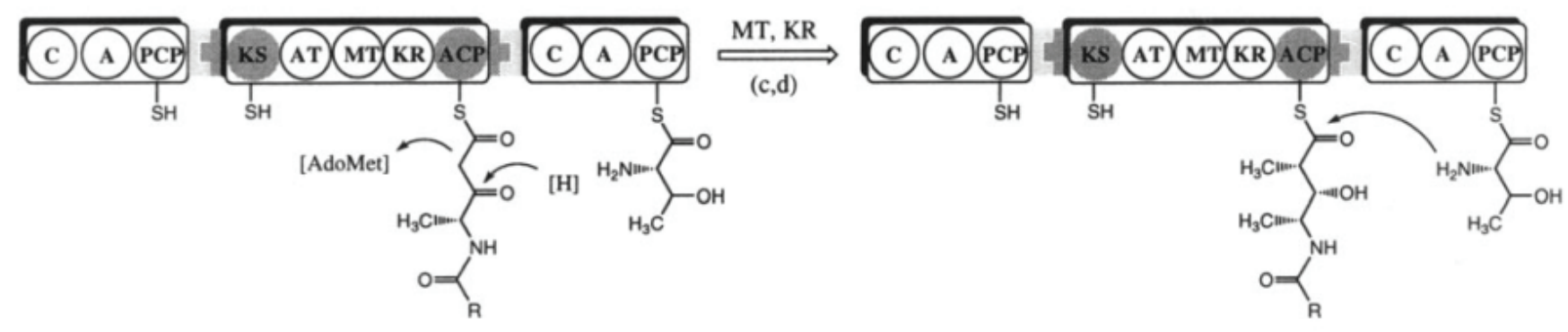

"peptide/polyketide"

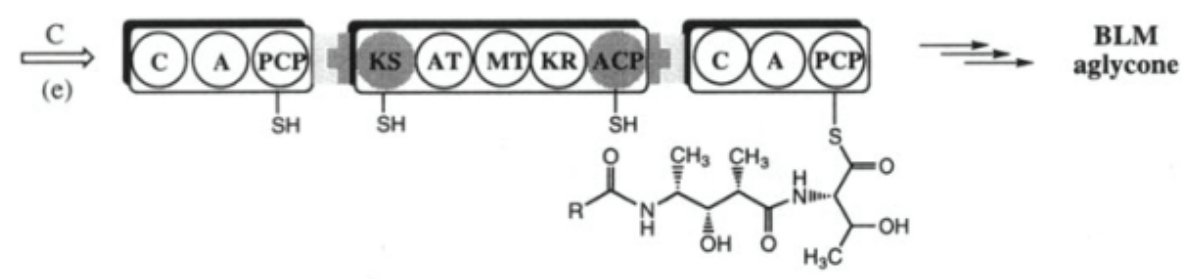

"peptide/polyketide/peptide"

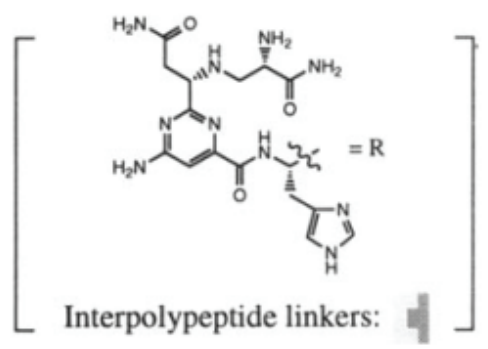

Figure 4 Schematic representation of the BImIX/BImVIII/BImVII system as a model for channeling the growing intermediate between NRPS and PKSmodule s or between PKSand NRPSmodules for biosynthesis of hybrid peptide-polyketide natural products. The KS and ACP domains of BIm VIII and the putative interpolypeptide linkers between BImIX/BImVIII and BImVIII/BImVII are shaded to emphasize their roles in facilitating interactions between NRPSand PKSto constitute a functional hybrid NRPS-PKSsystem.

mains of the PKS and NRPS modules, respectively. While the acceptor site substrate of amino acyl-S-PCP remains the same, the $\mathrm{C}$ domain in a hybrid PKS/ NRPS has to differentiate the donor site substrate of acyl-S-ACP for polyketide/peptide biosynthesis from peptidyl-S-PCP for peptide biosynthesis.

In polyketide biosynthesis, the $\mathrm{C}-\mathrm{C}$ bond formation is mediated by the KS domain that catalyzes (1) the transfer of the acyl-S-ACP from the upstream module, the donor site substrate, to the active site cysteine of KS and (2) the decarboxylative condensation between the resulting acyl-S-KS and its cognate malonyl-S-ACP, the acceptor site substrate [2- 4]. (Both "donor site" and "acceptor site" substrates for PKS are defined in a mechanistic analogy to NRPS [1].) In hybrid peptide/polyketide biosynthesis, however, the KS domain of the hybrid NRPS/PKS mediates the same $\mathrm{C}-\mathrm{C}$ bond formation but by catalyzing transfer of the peptidyl-S-PCP from the upstream NRPS module to the active site cysteine of KS to form a peptidyl-S-KS species. The latter then undergoes similar decarboxylative condensation with its cognate malony-S-ACP to yield the hybrid peptide/polyketide product (Figure 1B). According to this model, the critical domains for functional in- teraction in a hybrid NRPS/PKS should be the PCP and KS domains of the NRPS and PKS modules, respectively. While the acceptor site substrate of malonyl-S-ACP remains the same, the KS domain in a hybrid NRPS/PKS has to differentiate the donor site substrate of peptidyl-S-PCP for hybrid peptide/polyketide biosynthesis from acyl-S-ACP for polykeptide biosynthesis.

Many bacterial genomes encode multiple peptide and/ or polyketide pathways, each of which is composed of multiple PKS or NRPS modules. However, there is no evidence for crosstalk either among NRPS or PKS modules or between NRPS or PKS modules that belong to different peptide or polyketide pathways. In fact, NRPS or PKS modules constitute the NRPS or PKS megasynthetase with a precise selectivity, as evidenced by the fact that most of the NRPS or PKS pathways often produce only one major peptide or polyketide product out of a myriad of possibilities. These observations suggest the existence of extensive intermodular communication among modules that constitute the NRPS or PKS megasynthetases, raising an interesting question of how NRPS and PKS modules communicate to form hybrid NRPS-PKS 
systems. BlmIX/BlmVIII/BlmVII constitutes a natural hybrid NRPS/PKS/NRPS system, combining features of both hybrid NRPS/PKS and PKS/NRPS systems. We, therefore, reasoned that a detailed comparison of BlmIX/BlmVIII/BlmlVII with NRPS and PKS modules in nonhybrid systems could shed light on the mechanism for NRPS and PKS modules to constitute either hybrid NRPS/PKS or PKS/NRPS systems.

For BlmIX/BlmVIII, we found that the PCP domain of BlmIX seems to have no relevant difference compared to those in nonhybrid NRPS systems. In contrast, the KS domain of BlmVIII is unique in comparison with those in nonhybrid PKS systems. Upon phylogenic analysis, the KS domain of BlmVIII can be grouped into a subfamily of KSs from all other known hybrid NRPS/PKS systems regardless of their origins. Therefore, the BlmVIII PKS may have evolved a KS domain with an altered donor site substrate specificity to adapt the BlmIX-bound peptidyl-S-PCP intermediate (Figure 4, step a). Once the peptidyl intermediate is transferred to the active site cysteine of the KS domain, BlmVIII appears to utilize the same catalytic sites conserved in all other PKS domains to execute the decarboxylative condensation with the acceptor substrate of malonyl-S-ACP (Figure 4, step b) and the associated modifications (Figure 4, steps $\mathrm{c}$ and d) to yield the resultant peptide/polyketide product $[7,8]$.

For BlmVIII/BlmVII, we found that the ACP domain of BlmVIII is more similar to PCPs than to ACPs but the C domain of BlmVII shows no unique features compared to those in nonhybrid NRPSs [7,8]. These findings are in accordance with (1) the proposed function for the carrier proteins of both PKS and NRPS as workstations without any selectivity $[11,16]$ and (2) the low selectivity of the NRPS C domain toward the donor site substrate [1]. Physical proximity of the active sites may be sufficient for the $\mathrm{C}$ domain to accept the donor site substrate of acyl-S-ACP, instead of peptidyl-S-PCP, and to catalyze its elongation with the acceptor site substrate of amino acyl-S-PCP to afford the hybrid polyketide/peptide product (Figure 4, step e) $[7,8]$.

Intermodular communications in PKS have been attributed to either intermodular linkers that exist between modules within a protein or interpolypeptide linkers that exist between modules residing on separate proteins $[13,14]$. Polyketide chain transfer between modules appears to be permissive as long as the linkers can provide the connectivity between the adjacent modules [14]. Inspired by the linker hypothesis for PKS, we carried out similar sequence analyses for hybrid NRPS-PKS systems with the intention of identifying analogous linkers [8]. It is hoped that the specificities of the linkers between nonhybrid NRPS or PKS systems and hybrid NRPSPKS systems may provide insight into the evolution of NRPS and PKS modules into hybrid NRPS-PKS systems. We have indeed identified putative interpolypeptide linkers for both hybrid NRPS/PKS and hybrid PKS/NRPS systems. They are located at the C-termini of the NRPS proteins and N-terminal of the PKS proteins for hybrid NRPS/PKS systems and the C-termini of the PKS proteins and N-termini of the NRPS proteins for PKS/NRPS systems. The linkers vary from 5 to 76 amino acids and are rich in acidic residues [8]. It is tempting to pro- pose that these linkers may play a critical role in protein-protein recognition to constitute a functional hybrid NRPS-PKS system, as depicted for BlmIX/ BlmVIII/BlmVII (Figure 4). However, the latter conclusion is based purely on sequence analysis and has to be assessed experimentally in the future.

\section{Posttranslational modification of the carrier pro- teins of hybrid NRPS-PKS systems by a PPTase with broad substrate specificity}

For NRPS and PKS to be functional, their carrier proteins must be converted from the apo forms to the holo forms by covalent attachments of the 4'-phosphopantetheine group to an absolutely conserved serine residue. This posttranslational modification is catalyzed by a superfamily of enzymes known as PPTases that derive the 4'-phosphopantetheine group from coenzyme A (CoA) (Figure 1A) [20,31]. Most of the PPTases characterized to date exhibit high specificity toward the carrier protein substrates, leading to the proposal that each 4'phosphopantetheine-requiring pathway has its own posttranslational modifying PPTase activity [31]. However, a functional hybrid NRPS-PKS system requires a PPTase that is capable of modifying the apo forms of both PCP and ACP into their holo forms. Because all PPTases characterized to date are associated with fatty acid, polyketide, or peptide biosynthetic pathways, we were particularly interested in finding out if a PPTase from organisms that produce hybrid peptidepolyketide natural products exhibits relaxed specificity toward both ACPs and PCPs.

Most of the PPTases known to date were identified either by association with a peptide or polyketide biosynthetic gene cluster or by whole genome sequencing. Sequence analysis of the blm biosynthetic gene cluster, however, failed to reveal an associated PPTase gene, suggesting that the blm NRPS and PKS genes are not clustered with their PPTase gene [7]. To clone a PPTase gene from S. verticillus, we developed a PCR method using primers designed according to two conserved motifs among PCP-specific PPTases of actinomycete origin. The cloned Svp PPTase gene is mapped to an independent locus not clustered with any of the NRPS or PKS loci previously known to $S$. verticillus, including the blm hybrid peptide-polyketide gene cluster. The Svp protein was overproduced in E. coli, purified to homogeneity, and determined to be a monomer in solution. We have been able to show that Svp can efficiently 4'-phosphopantetheinate both type I and type II ACPs and PCPs from either S. verticillus or other Streptomyces species [23]. As compared to Sfp, the only other PPTase known to accept ACPs, ArCPs, and PCPs from various organisms, Svp displays a similar catalytic efficiency for PCPs but a 345-fold increase in catalytic efficiency for the tested ACP. Sfp, isolated from Bacillus subtilis, is clustered with the biosynthetic genes for the nonribosomal peptide antibiotic surfactin [21]. Sfp has been called a "promiscuous" PPTase, because (1) all other known PPTases, associated with fatty acid, peptide or polyketide biosynthetic gene clusters, are specific for their respective ACPs or PCPs, and (2) surfactin biosynthesis does not require the participation of an $\mathrm{ACP}$ 
[20-22,31]. In contrast, we argued that the broad substrate specificity of Svp toward various carrier proteins is an intrinsic property for PPTases that are generally responsible for secondary metabolite biosynthesis. These PPTases are nonessential for the producing organisms and, therefore, may evolve new or broad substrate specificity toward their carrier proteins including both PCPs and ACPs for hybrid peptide- polyketide biosynthesis [23]. Although we have shown that $S$. verticillus appears to have evolved a single PPTase, Svp, to posttranslationally modify the BLM hybrid NRPS-PKS system, it is yet to be determined if a PPTase with a broad carrier protein specificity is a general solution to provide functional hybrid NRPS-PKS systems for hybrid peptide-polyketide natural product biosynthesis.

\section{Concluding remarks}

BLM is an example of hybrid peptide-polyketide natural products. The peptide/polyketide/peptide backbone of the BLM aglycone is assembled by the BLM megasynthetase that consists of both NRPS and PKS modules. BlmIX/ BlmVIII /BlmVII constitute a natural hybrid NRPS/PKS and PKS/NRPS system, catalyzing the elongation of a peptidyl intermediate with a short carboxylic acid and a polyketide intermediate with an amino acid. This system therefore serves as a model for both hybrid NRPS/ PKS and PKS/NRPS systems. While biochemical data are still lacking, sequence analysis and functional comparison of domains and modules of BlmIX/BlmVIII /BlmVII with those of nonhybrid NRPS and PKS systems are beginning to shed light on hybrid peptidepolyketide biosynthesis. The same catalytic sites appear to be conserved in both hybrid NRPS-PKS and nonhybrid NRPS or PKS systems, with the exception of the KS domains in the hybrid NRPS/PKS systems that are unique. Specific linkers, confirmed for PKS $[13,14]$, and proposed for NRPS and hybrid NRPS-PKS systems [8], may play a critical role in intermodular communication to facilitate the transfer of the growing intermediates between the interacting NRPS and/or PKS modules. In order to be functional, hybrid NRPS-PKS systems require PPTases that should be capable of posttranslationally modifying the apo forms of both PCP and ACP into their holo forms. For the BLM megasynthetase, this modification has been accomplished by evolving a single PPTase, Svp, with a broad substrate specificity toward the apo forms of both ACPs and PCPs. These findings should now help us to formulate research ideas and experimental strategies for studying the biosynthesis of hybrid peptide- polyketide natural products.

\section{Acknowledgements}

This work was supported in part by an Institutional Research Grant from the American Cancer Society and the School of Medicine, University of California, Davis; the National Institutes of Health grant AI40475 and the Searle Scholars Program/The Chicago Community Trust. C.S. was supported in part by a postdoctoral fellowship from the Spanish Ministry of Education and Culture; D.J.E was supported in part by the
National Institutes of Health training grant T32GM07377; and J.M.M was supported in part by the Biotechnology Training Grant from the University of California, Davis.

\section{References}

1 Belshaw PJ, CT Walsh and T Stachelhaus. 1999. Aminoacyl-CoAs as probes of condensation domain selectivity in nonribosomal peptide synthesis. Science 284: 486-489.

2 Cane DE. 1997. A special thematic issue on polyketide and nonribosomal polypeptide biosynthesis. Chem Rev 97: 2463-2706.

3 Cane DE and CT Walsh. 1999. The parallel and convergent universes of polyketide synthases and nonribosomal peptide synthetases. Chem Biol 6: R319- R325.

4 Cane DE, CT Walsh and C Khosla. 1998. Harnessing the biosynthetic code: Combinations, permutations, and mutations. Science 282: 6368.

5 Challis GL, J Ravel and CA Townsend. 2000. Predictive, structurebased model of amino acid recognition by nonribosomal peptide synthetase adenylation domains. Chem Biol 7: 211 - 224.

6 Du L, M Chen, C Sánchez and B Shen. 2000. An oxidation domain in the BlmIII non-ribosomal peptide synthetase probably catalyzing thiazole formation in the biosynthesis of the antitumor drug bleomycin in Streptomyces verticillus. FEMS Microbiol Lett 289: 171-175.

7 Du L, C Sánchez, M Chen, DJ Edwards and B Shen. 2000. The biosynthetic gene cluster for the antitumor drug bleomycin from Streptomyces verticillus ATCC15003 supporting functional interactions between nonribosomal peptide synthetases and a polyketide synthase. Chem Biol 7: 623-642.

8 DuL,CSánchez and B Shen. 2001. Hybrid peptide- polyketide natural products: Biosynthesis and prospects towards engineering novel molecules. Met Eng 3: 78-85.

9 Du L and B Shen. 1999. Identification of a type II peptidyl carrier protein from the bleomycin producer Streptomyces verticillus ATCC 15003. Chem Biol 6: 507-517.

$10 \mathrm{Du}$ L and B Shen. 2001. Biosynthesis of hybrid peptide- polyketide natural products. Curr Opin Drug Discovery Dev 3: 215- 228.

11 Ehmann DE, CA Shaw-Reid, HC Losey and CT Walsh. 2000. The EntF and EntE adenylation domains of Escherichia coli enterobactin synthetase: Sequestration and selectivity in acyl-AMP transfers to thiolation domain cosubstrates. Proc Natl Acad Sci USA 97: 25092514.

12 Fujii A. 1979. Biosynthetic aspects of bleomycin- phleomycin group antibiotics. In: Hecht SM (Ed), Bleomycin: Chemical, Biochemical, and Biological Aspects. Springer-Verlag, New York, pp. 75-91.

13 Gokhale RS and C Khosla. 2000. Role of linkers in communication between protein modules. Curr Opinion Chem Biol 4: 22-27.

14 Gokhale RS, SY Tsuji, DE Cane and C Khosla. 1999. Dissecting and exploiting intermodular communication in polyketide synthases. Science 284: 482- 485.

15 Haydock SF, PE Leadlay, et al. 1995. Divergent sequence motifs correlated with the substrate specificity of (methyl) malonyl-CoA:acyl carrier protein transacylase domains in modular polyketide synthase. FEBS Lett 374: 246- 248.

16 Keating TA, ZC Suo, DE Ehmann and CT Walsh. 2000. Selectivity of the yersiniabactin synthetase adenylation domain in the two-step 
process of amino acid activation and transfer to a hole-carrier protein domain. Biochemistry 39: 2297-2306.

17 Kennedy J, K Auclair, SG Kendrew, C Park, JC Vederas and CR Hutchinson. 1999. Modulation of polyketide synthase activity by accessory proteins during lovastatin biosynthesis. Science 284: 13681372.

18 König A, PE Leadlay, et al. 1997. The pipecolate-incorporating enzyme for the biosynthesis of the immunosuppressant rapamycin- nucleotide sequence analysis, disruption and heterologous expression of rapP from Streptomyces hygroscopicus. Eur J Biochem 247: 526534.

19 Konz D and MA Marahiel. 1999. How do peptide synthetases generate structural diversity? Chem Biol 6: R39- R48.

20 Lambalot RH, CT Walsh, et al. 1996. A new enzyme superfamilythe phosphopantetheinyl transferases. Chem Biol 3: 923-936.

21 Nakano MM, N Corbell, J Besson and P Zuber. 1992. Isolation and characterization of sfp: A gene that functions in the production of the lipopeptide biosurfactant, surfactin, in Bacillus subtilis. Mol Gen Genet 232: 313-321.

22 Reuter K, MR Mofid, MA Marahiel and R Ficner. 1999. Crystal structure of the surfactin synthetase-activating enzyme Sfp: A prototype of the 4'-phosphopantetheinyl transferase superfamily. EMBO $J \mathbf{1 8}$ : 6823-6831.

23 Sánchez C, L Du, DJ Edwards and B Shen. 2001. Cloning and characterization of a phosphopantetheinyl transferase from Streptomyces verticillus ATCC15003, the producer of the hybrid peptidepolyketide antitumor drug bleomycin. Chem Biol 8: 725- 738 .
24 Shen B, L Du, C Sánchez, M Chen and DJ Edwards. 1999. Bleomycin biosynthesis in Streptomyces verticillus ATCC15003: A model of hybrid peptide and polyketide biosynthesis. Bioorg Chem 27: 155171.

25 Stachelhaus T, HD Mootz, V Bergendahl and MA Marahiel. 1998. Peptide bond formation in nonribosomal peptide biosynthesis: Catalytic role of the condensation domain. J Biol Chem 273: 2277322781.

26 Stachelhaus T, HD Mootz and MA Marahiel. 1999. The specificityconferring code of adenylation domains in nonribosomal peptide synthetases. Chem Biol 6: 493- 505.

27 Takita T. 1984. The bleomycins: Properties, biosynthesis, and fermentation. Drugs Pharm Sci 22: 595- 603.

28 Takita T and Y Muroka. 1990. Biosynthesis and chemical synthesis of bleomycin. In: Kleinkauf $\mathrm{H}$ and $\mathrm{H}$ von Döhren (Eds), Biochemistry of Peptide Antibiotics: Recent Advances in the Biotechnology of $\beta$-Lactams and Microbial Peptides. W de Gruyter, New York, pp. 289-309.

29 Umezawa H. 1980. Recent progress in bleomycin studies. In: Cassady JN and JD Douros (Eds), Anticancer Agents Based on Natural Product Models. Academic Press, New York, pp. 147-166.

30 von Döhren H, R Dieckmann and M Pavela-Vrancic. 1999. The nonribosomal code. Chem Biol 6: R273-R279.

31 Walsh CT, AM Gehring, PH Weinreb, LEN Quadri and RS Flugel. 1997. Post-translational modification of polyketide and nonribosomal peptide synthases. Curr Opin Chem Biol 1: 309- 315. 